\title{
COMPARISON OF HYPERBOLIC AND EXTREMAL LENGTHS
}

\author{
BERNARD MASKIT ${ }^{1)}$
}

Let $S$ be a hyperbolic Riemann surface of finite type (that is, $S=U / G$, where $U$ is the upper half-plane and $G$ is a finitely generated, torsion free Fuchsian group), and let $w$ be a hyperbolic simple loop on $S$ (that is, $w$ is a simple loop on $S$, and $w$ is represented by a hyperbolic element $A$ in $G$ ). There are two natural notions of length for such a loop: first, there is the hyperbolic length $l$ of the shortest geodesic freely homotopic to $w$ on $S$, and second, there is the extremal length $m$ of the family of loops freely homotopic to $w$ on $S$. The purpose of this note is to give some comparisons between these two notions of length.

When we need to emphasize the dependence of say $l$ on $w$, or $A$, or $S$, we will write $l(w)$, or $l(A)$, or $l(w, S)$.

The proofs all take place in the context of a non-elementary finitely generated Fuchsian group; the group may have torsion. All the results are easily seen to be equally valid for elementary Fuchsian groups.

In general, we say that a set $X \subset U$ is precisely invariant under the element $A \in G$ if $A(X)=X$, and $B(X) \cap X=\emptyset$ for all $B$ in $G$ which are not powers of $A$.

We say that the hyperbolic element $A \in G$ is strictly simple if the axis $L_{A}$ of $A$ is precisely invariant under $A$ in $G$. In particular, if $A$ is strictly simple, then there are no fixed points of elliptic elements of $G$ lying on $L_{A}$.

For any hyperbolic element $A$ of $G$, we define $l=l(A)$ to be the geodesic length of $A$; that is, $A$ is conjugate in $\operatorname{PSL}(2, R)$ to a unique element of the form $z \rightarrow e^{l} z$, $l>0$; equivalently, $|\operatorname{tr}(A)|=2 \cosh (l / 2)$. If $G$ is torsion free, this definition agrees with that of the first paragraph.

We let $w$ be the projection of $L_{A}$ on $U / G$, so that $w$ is a geodesic. Let $U^{\prime}$ be $U$ with all fixed points of elliptic elements of $G$ deleted, and let $S^{\prime}=U^{\prime} / G$. Then $m=$ $m(A)$ is the extremal length of the family of loops freely homotopic to $w$ on $S^{\prime}$.

We normalize $G$ so that $A(z)=e^{l} z, l>0$. We denote the projection from $U$ to $S$, or from $U^{\prime}$ to $S^{\prime}$, by $p: U \rightarrow S$.

A topological collar about $w$ is a subsurface $S_{0}$ of $S^{\prime}$, containing $w$, where $S_{0}$ is topologically an (open) annulus.

1) Research supported in part by NSF Grant MCS 8102621 
A topological collar about $L_{A}$ is a set $X$, containing $L_{A}$, which is precisely invariant under $A$ in $G$. A topological collar about $L_{A}$ of the form

$$
\left\{\pi / 2-\theta_{1}<\arg z<\pi / 2+\theta_{2}\right\}, 0 \leqq \theta_{1}, \theta_{2} \leqq \pi / 2,
$$

is a collar about $L_{A}$ of angle width $\theta=\theta_{1}+\theta_{2}$.

Proposition 1. If there is a collar about $L_{A}$ of angle width $\theta$, then

$$
m \theta \leqq l ;
$$

in any case,

$$
l \leqq m \pi .
$$

Proof. Let $T$ be the collar about $L_{A}$ of angle width $\theta$. Then $f(z)=\log (-i z)$, $f(i)=0$, maps $T$ onto a strip $V$ of height $\theta$, where $V$ is invariant under $H=\{z \rightarrow$ $z+l Z$ \}. The extremal length $m(w, p(T))$ is the extremal length of the family of curves connecting a point $z$ to $z+l$ in $V / H$; it is well known that the extremal length of this family is $l / \theta[2$, p. 12]. We now obtain inequality (1) from $m(w, S) \leqq$ $m(w, p(T))=l / \theta$.

Inequality (2) was proved in [7], but the statement there has the constant $2 \pi$ rather than $\pi$. For the convenience of the reader, we reprove it. Let $T$ be any topological collar about $L_{A}$. Then using the same function $f(z)=\log (-i z), f(T)$ is a topological strip invariant under $H$. We can estimate $m(w, p(T))$ by using the Euclidean metric in $f(T)$. We observe as above that the length of any curve is at least $l$, and since any vertical line intersects $f(T)$ in a set of measure at most $\pi$, the area of $f(T) / H$ is at most $\pi l$. Hence, $m(w, p(T)) \geqq l^{2} / \pi l=l / \pi$. It was shown by Jenkins [5] that the infimum of $m\left(w^{\prime}, S_{0}^{\prime}\right)$, where the infimum is taken over all topological collars $S_{0}^{\prime}$ about loops $w^{\prime}$, freely homotopic to $w$ on $S^{\prime}$, is in fact a minimum, and this minimum value is $m\left(w, S^{\prime}\right)$. Inequality (2) now follows.

The loop $w$ is called a boundary loop if $w$ divides $S$ into two subsurfaces and one of them is topologically an annulus. It is immediate that every boundary loop has a collar of angle width at least $\pi / 2$.

Corollary 1. If $w$ is a boundary loop, then $m \pi / 2 \leqq l \leqq m \pi$.

Our next proposition is a version of the collar lemma; other versions appear in Keen [6], Matelski [8], Buser [3], Randol [9], Abikoff [1], and Halpern [4].

Proposition 2. If $A(z)=e^{l} z$ is strictly simple, then $L_{A}$ has a collar of angle width $\theta$, where $\sin \theta / 2=e^{-l / 2}$. Further, if $B \in G$ is also strictly simple, where $p\left(L_{A}\right) \cap p\left(L_{B}\right)=\emptyset$, then these collars about $L_{A}$ and $L_{B}$ are disjoint.

Proof. Let $L_{B}$ be a hyperbolic axis in $G$, where $B$ represents a strictly simple loop $v$ in $U / G$, and either $v=w$, or $v$ is disjoint from $w$; we are primarily interested in the former case where $B=C \circ A \circ C^{-1}$, for some $C$ in $G$. Let $x$ and $y$ be the end- 
points of $L_{B}$; we can assume without loss of generality that $0<x<y$. Let $L$ be the hyperbolic line with endpoints $x$ and $e^{l} x=A(x)$. Since no translate of $L_{B}$ can cross $L_{B}, y \leqq e^{l} x$, and so $d\left(L_{A}, L\right) \leqq d\left(L_{A}, L_{B}\right)$, where $d(\cdot, \cdot)$ denotes hyperbolic distance. Let $M$ be the ray through the origin which is tangent to $L$. We write $M=$ $\{\arg z=\varphi\}$, and we observe that

$$
\sin \varphi=\left(e^{l} x-x\right) /\left(e^{l} x+x\right)=\tanh l / 2 .
$$

We note that $d\left(L_{A}, L\right)=d\left(L_{A}, M\right)$, and we choose $M^{\prime}$ to be that ray through the origin so that $d\left(M^{\prime}, L_{A}\right)=d\left(M^{\prime}, M\right)$. We write $M^{\prime}=\left\{\arg z=\pi / 2-\theta_{1}\right\}$, and we observe that we have chosen $M^{\prime}$ so that $L_{A}$ has a collar of angle width $2 \theta_{1}=\theta$.

An easy computation shows that

and

$$
d\left(L_{A}, M\right)=\log (\csc \varphi+\cot \varphi),
$$

$$
d\left(L_{A}, M^{\prime}\right)=\log \left(\csc \left(\pi / 2-\theta_{1}\right)+\cot \left(\pi / 2-\theta_{1}\right)\right) .
$$

Hence

$$
\left(1+\sin \theta_{1}\right) / \cos \theta_{1}=((1+\cos \varphi) / \sin \varphi)^{1 / 2} .
$$

We set the right hand side of (4) equal to $Q$; note that $\sin \theta_{1}>0$, and solve (4) for $\sin \theta_{1}$. We obtain

$$
\sin \theta_{1}=\frac{Q^{2}-1}{Q^{2}+1} .
$$

We combine (3), (4), and (5) to obtain

$$
\sin \theta_{1}=\frac{1+\cosh l / 2-\sinh l / 2}{1+\cosh l / 2+\sinh l / 2}=e^{-l / 2} .
$$

Once we have chosen $A$ in $G$, we can of course consider $l$ and $m$ as functions on the Teichmuller space $T(G)$. The remainder of our note takes place in this setting.

Corollary 2. The lengths $l$ and $m$ go to zero together, and $\lim _{l \rightarrow 0} l / m=\pi$.

Corollary 3. $m \leqq(1 / 2) l e^{l / 2}$.

Proof. We know from Proposition 2 that $L_{A}$ has a collar of angle width $\theta$, where $\sin \theta / 2=e^{-l / 2}$. Thus $\theta / 2 \geqq \sin \theta / 2=e^{-l / 2}$; hence by Proposition $1,2 m e^{-l / 2} \leqq l$, or

$$
m \leqq(1 / 2) l e^{l / 2} \text {. }
$$

We conclude this note with an example showing that the estimate (6) is not very far from being sharp.

For each $\alpha, 0<\alpha<\pi / 2$, we write down the Fuchsian group $G_{\alpha}$, generated by

$$
A_{\alpha}=\left[\begin{array}{ll}
\csc \alpha & \cot \alpha \\
\cot \alpha & \csc \alpha
\end{array}\right], \quad B_{\alpha}=\left[\begin{array}{cr}
\sec \alpha & i \tan \alpha \\
-i \tan \alpha & \sec \alpha
\end{array}\right]
$$


Observe that $A_{\alpha}$ has its fixed points at \pm 1 , while $B_{\alpha}$ has its fixed points at $\pm i$.

A fundamental domain $D_{\alpha}$ for $G_{\alpha}$ can be obtained by drawing the four hyperbolic lines with endpoints $e^{i \alpha}$ and $-e^{-i \alpha},-e^{-i \alpha}$ and $-e^{i \alpha},-e^{i \alpha}$ and $e^{-i \alpha}$, and $e^{-i \alpha}$ and $e^{i \alpha}$; see Figure 1 . We see at once that $G$ has signature $(1,1 ; \infty)$.

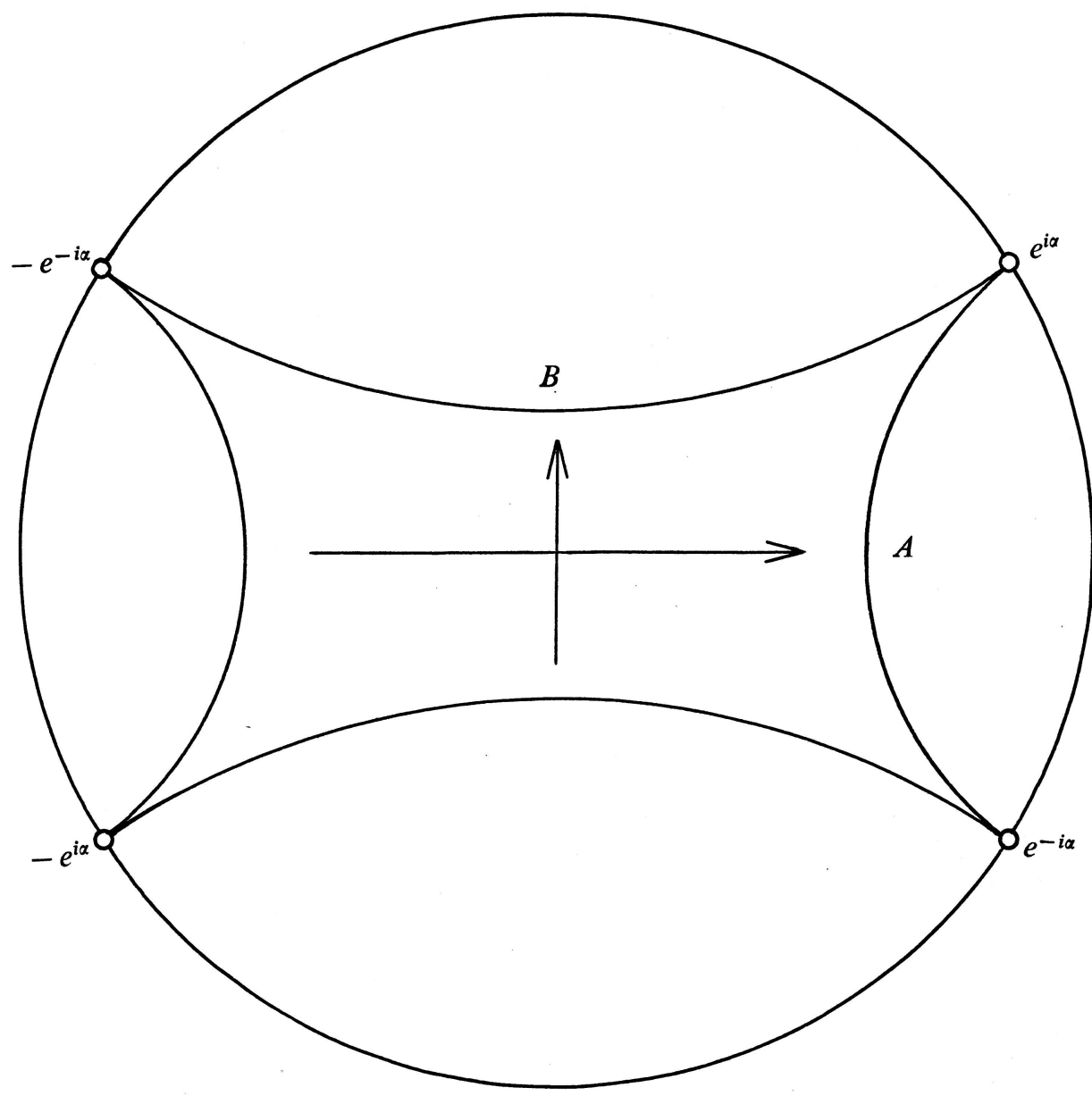

Figure 1

The reflection $j(z)=\bar{z}$ commutes with $A=A_{\alpha}$, and conjugates $B=B_{\alpha}$ into $B^{-1}$. Hence the elliptic modulus of the torus $U / G$ with the generators $A$ and $B$ is pure imaginary. Since the sides of $D_{\alpha}$ are fixed point sets of reflections in the group generated by $G_{\alpha}, j$, and the reflection $z \rightarrow-\bar{z}$, the covering map $\varphi$, from $U$ onto the plane punctured at the lattice points, maps $D_{\alpha}$ onto a rectangle, and conjugates $A$ and 
$B$ into translations in the plane. We conclude that the extremal length of the family of paths joining a point $z$ on the boundary of $D_{\alpha}$ to $A(z)$ (or $z$ to $B(z)$ ), is equal to the extremal length of the family of paths joining opposite sides of the rectangle, $\varphi\left(D_{\alpha}\right)$. In particular $m(A)=1 / m(B)$.

Let $\theta_{B}$ be the angle width of the largest collar about $L_{B}$. Then,

$$
m(A)=1 / m(B) \geqq \theta_{B} / l(B)=2 l(B)^{-1} \arcsin \left(e^{-l(B) / 2}\right) \geqq 2 l(B)^{-1} e^{-l(B) / 2} .
$$

An easy computation shows that $l(B)=2 \log (\sec \alpha+\tan \alpha)$, and so

$$
\begin{aligned}
m(A) & \geqq 1 /(\sec \alpha+\tan \alpha) \log (\sec \alpha+\tan \alpha) \\
& \geqq 1 /(\sec \alpha+\tan \alpha)(\sec \alpha+\tan \alpha-1) .
\end{aligned}
$$

We rewrite the right hand side of the above as

$$
R=\cos ^{2} \alpha /(1+\sin \alpha)(1+\sin \alpha-\cos \alpha) .
$$

We note that $l(A)=2 \log (\csc \alpha+\cot \alpha)$, and we observe that

$$
\lim _{\alpha \rightarrow 0} \operatorname{Re}^{-l(A) / 2}=\lim _{\alpha \rightarrow 0} R /(\csc \alpha+\cot \alpha)=1 / 2 .
$$

We conclude that for every $\varepsilon>0$, we can find an $\alpha$ so that

$$
m(A) \geqq R \geqq(1 / 2-\varepsilon) e^{l(A) / 2} .
$$

Remark 1 . The requirement that $G$ be finitely generated was used only in Jenkins' theorem. Proposition 2 is valid for an arbitrary Fuchsian group. Proposition 1 is also valid in this more general context, provided one understands $m$ as the infimum of $m\left(w^{\prime}, S_{0}{ }^{\prime}\right)$, where $w^{\prime}$ is freely homotopic to $w$, and $S_{0}^{\prime}$ is any annulus containing $w^{\prime}$, and contained in $S_{0}$.

Remark 2. If $L_{A}$ has elliptic fixed points on it, but is otherwise simple, the results are slightly different. We outline these below.

Let $A$ be a hyperbolic element of the finitely generated Fuchsian group $G$, where for every $B \in G$, either $B\left(L_{A}\right)=L_{A}$, or $B\left(L_{A}\right) \cap L_{A}=\emptyset$. Assume that there is an element $E \in G$, where $E$ is not a power of $A$, and $E\left(L_{A}\right)=L_{A}$. Then $E$ is necessarily elliptic of order 2, and $p\left(L_{A}\right)$ is a path from one ramification point of order 2 to another. Call these ramification points $x$ and $x^{\prime}$, and let $w$ be a simple loop which separates $S^{\prime}$ into two subsurfaces, where one of these is a disc with the two punctures, $x$ and $x^{\prime}$. We have already defined $l(A)=l(w)$, and we set $m(A)=m(w)$.

We normalize $G$ so that $A(z)=e^{l} z$, and so that $E$ has fixed points at $\pm i$; then $A \circ E$ has its fixed points at $\pm i e^{l / 2}$.

If $\left\{\pi / 2-\theta_{1}<\arg z<\pi / 2+\theta_{2}\right\}$ is a collar about $L_{A}$ (i.e., it is precisely invariant under the stability subgroup of $L_{A}$ in $G$ ), then either $\theta_{1}=0$, or $\theta_{2}=0$; we assume without loss of generality that $\theta_{2}=0$.

Inequality (1) still holds, and inequality (2) can be replaced with

$$
l \leqq m \pi / 2 .
$$


The proof is the same, except that to prove ( $\left.2^{\prime}\right)$, observe that $f$ conjugates $E$ into the transformation $z \rightarrow-z$; hence if $k$ is the measure of the intersection of $f(T)$ with the vertical line $\operatorname{Re}(z)=a, a<l / 2$, and $k^{\prime}$ is the measure of the intersection of $f(T)$ with the line $\operatorname{Re}(z)=-a$, then $k+k^{\prime} \leqq \pi$.

If $w$ is a boundary loop, then $G$ is elementary, and $l=m \pi / 2$, as can be verified directly.

The proof of Proposition 2 is essentially unchanged, but the statement is different.

Proposition 2'. If A represents a simple loop $w$, and $L_{A}$ has elliptic fixed points on it, then $L_{A}$ has a collar of angle width $\theta$, where $\sin \theta=e^{-l / 2}$.

Corollary 2'. If $A$ is as in Proposition $2^{\prime}$, then $l$ and $m$ go to zero together and $\lim _{l \rightarrow 0} l / m=\pi / 2$.

Corollary 3'. If $A$ is as in Proposition $2^{\prime}$, then $m \leqq l e^{l / 2}$.

The group $G_{\alpha}$ has a $Z_{2}$ extension $H_{\alpha}$ obtained by adjoining the transformation $j(z)=-z$. Then $l\left(A_{\alpha}, H_{\alpha}\right)=l\left(A_{\alpha}, G_{\alpha}\right), \quad l\left(B_{\alpha}, H_{\alpha}\right)=l\left(B_{\alpha}, G_{\alpha}\right), \quad$ and $\quad m\left(A_{\alpha}, H_{\alpha}\right)=$ $=2 m\left(A_{\alpha}, G_{\alpha}\right), m\left(B_{\alpha}, H_{\alpha}\right)=2 m\left(B_{\alpha}, G_{\alpha}\right)$. The first two equalities are trivial, and the second two follow from the fact that the map $\varphi$ commutes with $j$, and $\varphi$ maps the axes of $A$ and $B$ onto Euclidean lines which are parallel to the sides and bisect the rectangle $\varphi\left(D_{\alpha}\right)$.

In this case we obtain that for $\alpha$ sufficiently small, $m\left(A, H_{\alpha}\right) \geqq(1-\varepsilon) e^{l\left(A, H_{\alpha}\right) / 2}$.

\section{References}

[1] Aвikoff, W.: The real analytic theory of Teichmüller space. - Lecture Notes in Mathematics 820. Springer-Verlag, Berlin-Heidelberg-New York, 1980.

[2] Ahlfors, L. V.: Lectures on quasiconformal mappings. - D. Van Nostrand Company, Inc., Princeton, New Jersey-Toronto-New York-London, 1966.

[3] Buser, P.: The collar theorem and examples. - Manuscripta Math. 25, 1978, 349-357.

[. t] Halpern, N.: A proof of the collar lemma. - Bull. London Math. Soc. 13, 1981, 141-144.

[5] Jenkins, J. A.: On the existence of certain general extremal metrics. - Ann. of Math. (2) 66, 1957, $440-453$.

[6] KeEn, L.: Collars on Riemann surfaces, discontinuous groups and Riemann surfaces. - Discontinuous groups and Riemann surfaces. Ann. of Math. Studies 79. Princeton University Press, Princeton, New Jersey, 1974, 263-268.

[7] Maskit, B.: Parabolic elements in Kleinian groups. - Ann. of Math. (2) 117, 1983, 659-668.

[8] MAtelski, J. P.: A compactness theorem for Fuchsian groups of the second kind. - Duke Math.

J. 43, 1976, 829-840.

[9] Randol, B.: Cylinders in Riemann surfaces. - Comment. Math. Helv. 54, 1979, 1-5.

State University of New York at Stony Brook

Department of Mathematics

Stony Brook, New York 11794

USA

Received 11 April 1983 